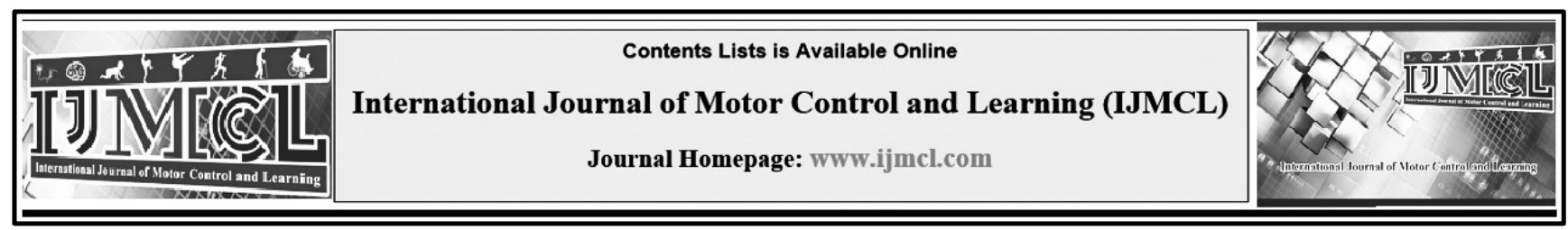

\title{
Improvement in Balance and Gait Speed of MS Patients under Challenge Point Framework
}

\author{
Masoud Zahiri ${ }^{\mathrm{a}^{*}}$, Shahzad Tahmasebi Boroujeni ${ }^{\mathrm{b}}$
}

a Ph.D. Student in Motor Control, University of Tehran, Tehran, Iran

${ }^{\mathrm{b}}$ Associate Professor in Motor Control, University of Tehran, Tehran, Iran

\begin{tabular}{|c|c|}
\hline Keywords & Abstract \\
\hline Challenge Point Framework & Background: Challenge Point Framework (CPF) is a model by which it is possible to \\
\hline Mobility & $\begin{array}{l}\text { enhance mobility learning through interference in practice conditions to adjust task } \\
\text { difficulty as a consequence of the interaction between skill levels of learner and the task }\end{array}$ \\
\hline Balance & difficulty. As the Multiple Sclerosis (MS) patients have trouble with balance control and \\
\hline Multiple Sclerosis & $\begin{array}{l}\text { walking, giving practice in the framework can help them cope with these movement } \\
\text { problems. In the previous studies, it was clear that the method was useful in Parkinson and } \\
\text { brain stroke patients, not in MS patients. Therefore, the purpose of this study is to address } \\
\text { the CPF to improve control balance and mobility of the MS patients. }\end{array}$ \\
\hline $\begin{array}{l}\text { Masoud, Zahiri, } \\
\text { Email: m.zahiry@ut.ac.ir }\end{array}$ & $\begin{array}{l}\left.\text { Method: We have randomly selected } 22 \text { individuals with MS ( } \mathrm{M}_{\mathrm{age}}=32 \pm 4.5_{\mathrm{yrs}}\right) \text { and } \\
\text { divided them into two groups of control and experiment with } 12 \text { interference sessions. We } \\
\text { employed Timed Up and Go (TUG) test to evaluate balance and } 25 \text {-foot walk test to } \\
\text { measure speed. }\end{array}$ \\
\hline Received: 2020/05/05 & Results: The results have indicated that the speed and balance of the patients in experiment \\
\hline $\begin{array}{l}\text { Accepted: } 2020 / 07 / 20 \\
\text { Published: } 2020 / 08 / 23\end{array}$ & $\begin{array}{l}\text { Conclusion: Therefore, the efficiency of CPF has been approved in gait speed and balance } \\
\text { of the MS patients. }\end{array}$ \\
\hline
\end{tabular}

\section{Introduction}

Multiple sclerosis, known as MS, is one of the most widespread chronic diseases with demyelination of nervous neurons. White matter in the nervous system is covered by many demyelinated axons that affect sense and movement functions. According to the studies, there are about 50000 patients with 9000 people recorded and the value is increasing (Ghasemi, Gorji, Ashtar, \& Ghasemi, 2015). The most widespread age of the disease is ranged from 20 to 40 (Lapierre, et al, 2016), i.e., the age with many social and family responsibilities for them. As a result, the disease endangers the useful human forces of the entire community. The symptoms of the disease are unknown and the patients experience a variety of physical and psychological disorders (Martino, Espay, Fasano, \& Morgante, 2016). The most common symptoms of the disease are non-normal walking, dysfunction in postural control and balance, muscle spasms, fatigue, spasticity, sensing disorders, vision problems, and postural stability disorders. These problems affect all the social and personal tasks of the patients. Thus, the MS is a very disabling disease (Blair et al., 2016).

The researches about the theories and model of learning have greatly developed in the recent years. 
For example, Challenge Point Framework (CPF) was introduced by Guadagnoli and Lee (2004). This is a theoretical view to understand the effects of practice variables in movement learning. In the framework, contextual interference variables and knowledge of result feedback have a powerful relationship with skill learning level and task difficulty of the learners. Indeed, this framework indicates several variables involved in learning as a single protocol(M. A. Guadagnoli \& Bertram, 2014; MA Guadagnoli \& Lee, 2004; Mark Guadagnoli \& Lindquist, 2007).

In CPF, it is stated that the learning is influenced by available information and the ability of the learner in interpretation and use of the information. The information is provided through nature of task or other persons (coach, peer, etc.) and has very high or negligible negative effects on the Learning. Therefore, the purpose of practice design is based on CPF principles, task difficulty level, and optimal challenge point for learners by controlling the information through manipulation of two important practice models, i.e., contextual interference and expanded feedback (Akizuki \& Ohashi, 2013).

To understand the concept of task difficulty, the variable is divided into two factors of functional difficulty and nominal difficulty. The task nominal difficulty reflects a fixed amount of difficulty about the performer and the environmental situations. Therefore, this can contain factors including cognitive and movement needs of the task. On the contrary, functional difficulty of task is influenced by some factors including practice positions and subject skill level. Thus, the main assumption of the $\mathrm{CPF}$ is that Learning is a problem-solving process and that memorized information is the basis of the Learning. Accordingly, in CPF the characteristics of the learners influence on how they respond the practice positions(M Guadagnoli \& Aylsworth, 2013; Onla-or \& Winstein, 2007). Based on the fundamentals of $\mathrm{CPF}$, the Learning has a direct relationship with the ability to access information and their interpretation according to functional difficulty of the task. It is supposed that information makes an involvement challenge in learners and when the information is proposed it gives an opportunity for Learning. Increase in task difficulty is followed by increase in potential information. As the functional difficulty is increased, the ability to interpret the information is also increased (Akizuki \& Ohashi, 2013). However, there is limitation in the ability to interpret the information. The information is controlled by information processing capacities and by the fact that if the capacities can change by practice. In $\mathrm{CPF}$, the assumptive points are considered for each individual with any skill level. These points, called optimal challenge points, are indicative of optimal values for interpretable information for Learning in an individual. In the optimal challenge points, there is an appropriate amount of accessible information necessary for suitable information. After this point, the accessible information will be highly increased and exceed the capacity of the individuals for efficient processing and interpretation. As a result, the potential advantages of Learning are decreased. In 
other words, even if adequate information is available, this does not mean increase in interpretable information. Thus, the learners cannot efficiently process the accessible information to improve their skill level. Therefore, the information processing system is broken and interrupts the Learning and performance(MA Guadagnoli \& Lee, 2004).

In the past decades, researchers have been seeking the practice situations and the processes that lead to increase in performance and facilitation of Learning and use of the basics of movement learning such as interference in information presentation, practice planning, and levels of physical aids. These are well considered in CPF. These can be optimal methods to retrain movement skills, preserve and conduct them in spinal and brain disorder diseases such as MS. Given that the practices based on CPF were useful in Parkinson (Onla-or \& Winstein, 2007) and brain stroke(Pollock, Boyd, Hunt, \& Garland, 2014), as confirmed in the previous studies, and that the main treatments of cognitive and physical disorders of the MS patients are through medicine, nutrition, and psychotherapy with no physical activities, particularly the practices appropriate to their body conditions, it is an important task to find the most appropriate practice protocol in terms of both practice method and duration. Therefore, the objective of this research is to apply CPF through purposeful interfering in four important Learning factors including learner skill level (control criterion for including MS patients in this research), frequency of feedback, contextual interference, and task difficulty.

\section{Method}

\subsection{Study design}

This study is a semi-experimental research with iterative measurement design. The applied research has been conducted in pretest-posttest method with control group to evaluate CPF-based practice.

\subsection{Participants}

Among the male MS patients with Expanded Disability Status Scale (EDSS) of 5.2 and 6.5 from MS forum of Ahvaz City, 30 persons accepted to participate in the research. They have put randomly in two groups of experimental and control. The criteria for inclusion to this research select the individuals with recorded MS for at least one year since diagnosis, not recurrence of the disease in the last month before the start of the experiment, having no other diseases, e.g., cardiovascular, respiration, cortical, and cognition disorders. The criteria to exclude the individuals out of the experiment are including not regular participation in practice sessions, recurrence of the disease during the experiment period, excess fatigue (so, it is not possible to continue the practices), participation in other sport practices, not attending in test sessions (Peruzzi, Cereatti, Croce, \& Mirelman, 2016). Three persons from control group have been excluded due to recurrence of the disease and using medicine and five persons have also excluded from experiment group due to not regular participation in practice sessions. Finally, 
22 patients as samples (10 samples in experiment and 12 samples in control) with average age of 32 years old have participated in this research. They were asked to read and sign the consent prior to this study.

\subsection{Measurement Instrument}

Timed Up and Go test (TUG) has been employed to evaluate balance and measure walking function and dynamic balance through the performances of standing, walking, rotating, and sitting. Each participant, sitting on an arm chair 45 $\mathrm{cm}$ high, on the word GO will stand up, walk on the line on the floor three meters away from the chair, turn around and walk back to the chair and sit down. With sitting of the participant, the test is ended. As the participant could not pass the end line, did not turn around, or sit down on the chair, the test has to be repeated. It is noteworthy that each test has been repeated three times, with resting to avoid tired, for each patient and the average value considered (Yim-Chiplis \& Talbot, 2000; Zampieri \& Di Fabio, 2008).

Timed 25 Foot Walk (T25-FW) is used to measure movement ability. Gait speed is accurate and valid criterion to measure movement ability of the MS patients (Sebastia, Sandroff, Learmonth, \& Motl, 2016). This is also one of the important tests to measure walking speed of the patients (Bethoux \& Bennett, 2011). The straight line devised next to wall to avoid the fear of the patients of having no staddle. The time it takes to the subjects pass the course is calculated as their record.

\subsection{Interference}

After the sample has been selected, detailed explanations have been presented about the goals of this research and the implementation stages. Then, after the subjects have agreed, balance and movement ability pretest have been conducted for them. Subsequently, the subjects of experiment group participated in four-week practice sessions. The interference contains 12 sessions of 45 minutes; 9 sessions for practice and 3 sessions for testing. The design of the practice and test sessions is based on previous research design (Pollock et al., 2014). During the practice, the subjects have performed two times the 30 tensional block of stepping reaction in each direction and with each foot. It has been totally 60 tensions with 10 tensions for each foot in each direction. After each 10 tensions with each foot, the subject has 1 minute of rest and after the first block finished, the subjects rested 5 minutes and continued the same procedure for the second foot. The first posttest has been taken at the end of the second week and the second posttest has taken at the end of fourth week. The task contains auto-Learning of stepping reaction in different directions. Thus, the subjects have been learnt to stand straight and make their body lax and lobby. Then, they have to blend themselves at the talus, in their feet, in three directions of forward, backward, and sides to their falling threshold. As soon as the subjects have felt that they are about to fall, the task is stopped and in case, they may take one or two steps around to control their balance. It is noteworthy that during the experiment the subject should not have blending in the knees 
pelvis and the stepping reaction is done just at the talus of feet (Karlsson \& Persson, 1997). One of the reasons to use the stepping reaction test is that in many daily body activities the talus is the first point that makes reaction to keep balance. The correct performance of the talus plays a key role in keeping the balance of body and postural situation in the patient ability. Contextual interference and gradual increase in functional difficulty of task have been done in the primary stages of the experiment as a block practice in order to optimize the Learning of movement skills. In the subsequent stages, random practice has been performed by the subjects to form movement learning (Pollock et al., 2014). The content and timing of the oral expanded feedback of the therapist have been designed to solve the primary problem and to prevent the possibility of interference in movement learning with development of skill level.

\subsection{Data Analysis}

For the analysis, we have used descriptive statistics of mean and standard deviation. ShapiroWilk test have been used to ensure normality of the data and Levene statistic to test homogeneity of variances. In inferential statistics, we have also used combined Analysis Of Variance (ANOVA) (two groups and three times), frequent analysis of variance, independent t-test, Bonferroni post hoc test (Verma, 2011). The data analyzed in SPSS 20 with confidence interval at $0.05(P \leq 0.05)$.

\section{Results}

The average values of mobility in control group are
$12.9 \pm 0.95$ in pretest, $12.72 \pm 0.75$ in the first posttest and $12.82 \pm 1.02$ in the second posttest. The values in experiment group are $12.96 \pm 0.96$ in pretest, 12. $2 \pm 0.9$ in the first posttest and $11.21 \pm 1.19$ in the second posttest (Table 1). The average and standard deviation values of balance in control group are $11.71 \pm 1.13$ in pretest, $11.77 \pm 0.89$ in the first posttest and $11.79 \pm 0.84$ in the second posttest. The values for experiment group are 11.89 \pm 1.18 in pretest, $10.94 \pm 0.64$ in the first posttest and $10.34 \pm 1.86$ in the second posttest (Table 1). The values indicate the advance of the interfered group. Given that the Mauchly's sphericity test is not significant $(P=0.22)$, the $\mathrm{F}$ indices related to sphericity is reported. Moreover, the Levene test for equality of variances has indicated that the $\mathrm{F}$ statistic is not significant for any of within group factors (pretest $P=0.89$, the first posttest 0.35 , and the second posttest $P=0.42$ ). Thus, homogeneity of variances is approved among the groups of independent variables.

The results of combined ANOVA have also indicated that the principal effects of balance measurement time $\quad\left(\mathrm{F}_{2,40}=11.68, \quad \mathrm{sig}=0.001\right.$, $\left.\eta^{2}=0.369\right)$ and mobility are significant $\left(F_{2,40}=18.47\right.$, sig $\left.=0.000002, \eta^{2}=0.48\right)$. In addition, the interaction of measurement time is significant with balance test group $\left(\mathrm{F}_{2,40}=14.63\right.$, sig $=0.001$, $\left.\eta^{2}=0.422\right) \quad$ and mobility $\quad\left(\mathrm{F}_{2,40}=15.93\right.$, $\left.\operatorname{sig}=0.000008, \eta^{2}=0.44\right)$. However, the principal effects of the group are not significant with balance $\left(F_{1,20}=3.63\right.$, sig $\left.=0.07, \eta^{2}=0.154\right)$ and mobility $\left(F_{1}\right.$, ${ }_{20}=3.32$, sig $=0.08, \eta^{2}=0.142$ ) (Table 3). Since the interactional effects are significant, we disregard 
the principal effects.

Table 1. The average and standard deviation values of TUG and gait speed in the participants.

\begin{tabular}{cccccc}
\hline Variable & Time & $\begin{array}{c}\text { Mean } \\
\text { control }\end{array}$ & $\begin{array}{c}\text { SD } \\
\text { control }\end{array}$ & $\begin{array}{c}\text { Mean } \\
\text { experimental }\end{array}$ & $\begin{array}{c}\text { SD } \\
\text { experimental }\end{array}$ \\
\hline & pre & 11.71 & 1.13 & 11.89 & 1.18 \\
Balance & Post 1 & 11.77 & 0.89 & 10.94 & 0.64 \\
Gait speed & Post 2 & 11.79 & 0.84 & 10.34 & 0.86 \\
& pre & 12.90 & 0.95 & 12.96 & 0.96 \\
& Post 1 & 12.72 & 0.75 & 11.21 & 0.90 \\
& Post 2 & 12.82 & 1.02 & & 1.19 \\
\hline
\end{tabular}

A within subject ANOVA design with iterative measurements on measurements stages has been used to determine the influence of CPF-based practices on the balance and gait speed in MS patients. The results of design have indicated that the CPF-based practices are able to improve the balance $\left(F_{2,18}=18.89\right.$, sig $\left.=0.001, \eta^{2}=0.677\right)$ and mobility $\left(\mathrm{F}_{2,18}=17.77\right.$, sig $\left.=0.001, \eta^{2}=0.664\right)$ of the MS patients. The results of Bonferroni post hoc test to determine the difference in measurement stages have also indicated that the balance from pretest to the first posttest $(P=0.033)$ and in second posttest is significant $(P=0.001)$. However, there is no significant difference between the first and second posttests $(P=0.08)$. The running speed from pretest to the first posttest is not significant $(P=0.079)$.

Table 2. Results of the combined ANOVA test.

\begin{tabular}{llccccc}
\hline Variable & & SS & DF & M S & F & $p$ \\
\hline Balance & TIME & 5.89 & 2 & 2.948 & 11.68 & $0.001^{*}$ \\
& Group & 8.009 & 1 & 8.009 & 3.63 & 0.07 \\
& Time*Group & 7.31 & 2 & 3.69 & 14.6 & $0.001^{*}$ \\
& TIME & 9.11 & 2 & 4.45 & 18.47 & $0.001^{*}$ \\
& Group & 7.75 & 1 & 7.75 & 3.32 & 0.08 \\
& Time* Group & 7.86 & 2 & 3.93 & 15.93 & $0.001^{*}$ \\
\hline
\end{tabular}

However, the difference is found between pretest and first posttest $(P=0.001)$ and between first posttests and second $(P=0.033)$. (Table 3$)$.
After the within group differences have been examined, independent $t$-test has been used to test between group differences of measurement stages (Table 4).

Table 3. The results of Bonferroni post hoc test to experiment group differences.

\begin{tabular}{lrrrrr}
\hline Variable & Time & & Mean & ST & p \\
\hline Balance & & Post 1 & 0.950 & 0.29 & $0.033^{*}$ \\
& Pre & Post 2 & 1.54 & 0.23 & $0.001^{*}$ \\
& Post 1 & Post 2 & 0.59 & 0.22 & 0.08 \\
\multirow{2}{*}{ Gait speed } & & Post 1 & 0.758 & 0.28 & 0.079 \\
& Pre & Post 2 & 1.750 & 0.28 & $0.001^{*}$ \\
& Post 1 & Post 2 & 0.992 & 0.31 & $0.033^{*}$ \\
\hline
\end{tabular}


Table 4. Results of independent t-test in balance and mobility in each stage of measurement.

\begin{tabular}{ccccc}
\hline Variable & Time & $\mathrm{t}$ & $\mathrm{P}$ & $\mathrm{df}$ \\
\hline \multirow{2}{*}{ Balance } & pre & 0.36 & 0.72 & 20 \\
& Post 1 & 2.83 & 0.024 & 20 \\
& Post 2 & 3.98 & 0.001 & 20 \\
Gait speed & pre & 0.155 & 0.878 & 20 \\
& Post 1 & 1.47 & 0.175 & 20 \\
& Post 2 & 3.38 & 0.003 & 20 \\
\hline
\end{tabular}

As it can be observed in Table 4, there is a significant difference in control and experiment groups in the first posttests $(P=0.024)$ and second posttest $(P=0.001)$ and there is no significant difference in mobility in second posttest $(P=0.003)$.

\section{Discussion}

The results of the present research have indicated that CPF-based four-week practice protocol has significant difference on balance control and mobility disorders of MS patients and that it can improve the balance and mobility in the patients. To follow the standards of CPF in the first posttest, the patients in terms of EDSS and the degree of disability in movement disorders have been considered as beginners in practice sessions. They performed the same practice of stepping reaction in a block form with a $30 \%$ to $50 \%$ feedback to regulate the practice difficulty according to the patient skill levels and low demand practice conditions. Given that this is the first research about the CPF in balance and gait speed of the MS patients, the results have been compared with the findings of the researches with CPF on other neurologic communities. The previous studies indicated that although low implicit interference and high frequency of feedback increase performance in acquisition stage, but combination of the two factors lead to decrease in healthy people with no physical disorder (Onla-or \& Winstein, 2007). However, these results are not confirmed in the patients with neural system disorders such as MS. For example, with the studies of Onla and Kroli (2008) in Parkinson patients and Polak et al (2014) on brain stroke patients and this research, this can be argued that high frequency feedback and low contextual interference (block practice) can improve the movement disorders in the MS patients. The results of this part can be compared with the studies of challenge point by Guadagnoli and Lee (2004). Based on CPF for the people with different levels of skill, low levels of contextual interference can provide the beginners with optimal challenge point in which better Learning can be occurred. Onla and Kroli (2008) investigated the influence of interference on practice condition (low demand and high demand practice conditions) and change in nominal difficulty of practice in two groups of healthy people and those with Parkinson. They concluded that in low demand practice conditions (random practice and high frequency feedback); Parkinson patients had better performance relative to healthy persons. In other words, when the functional difficulty was low, the persons with Parkinson showed better performance than the healthy persons. As the functional 
difficulty was moderate, the persons with Parkinson showed a decrease in performance. Therefore, added functional difficulty is a principal component in achieving optimal challenge point to promote better Learning in healthy persons compared with Parkinson persons (Krause et al., 2014; Martino et al., 2016).

The second posttest has been taken in high demand practice conditions with high functional difficulty (random practice and low added frequency of feedback, 20\%). The results, similar to the first posttest, represented a significant difference in control and experiment groups in balance and mobility. This is also typical of improvement in MS patients in this regard. This part also supports the $\mathrm{CPF}$ results in that based on $\mathrm{CPF}$, the random practice compared with blocked practice and decrease of frequency of feedback increases task difficulty and recognition. This can facilitate performance and Learning. The characteristics of learner and skill level can influence how they may response to the practice conditions. Therefore, it is possible that the practice conditions may be useful for healthy persons in Learning and performance but not useful for the persons with physical or neurotic disorders (Onla-or \& Winstein, 2007). Therefore, it seems that improvement in movement disorders of MS patients in the second posttest is likely resulted from the fact that the patient skill levels have also been improved in stepping reaction task though task difficulty increased. Thus, MS patients with different personal characteristics have experienced different tasks with high and low levels of task difficulty under particular practice conditions and certain practices. The findings is consistent with theory of dynamic systems about Learning parallel movement skills which state that the movement skills are under the influence of three factors of individual, task, and environment. With the principles and rules of $\mathrm{CPF}$, many researchers indicated that some factors including task difficulty and complexity, planning and primary learner skill level, and feedback type can influence Learning of movement skills(Hoang, Cameron, Gandevia, \& Lord, 2014; Ilett, Lythgo, Martin, \& Brock, 2015; Krause et al., 2014). Onla and Winstein (2008) stated that Parkinson patients compared with healthy persons had better transfer with increase in frequency of feedback and random practice in $100 \%$. They argued that the Learning mechanism in the persons with Parkinson disorder is not clear and may be different with that in healthy people. They also reported that normative frustrations play important role in change of task and feedback vertex of ramifications in marbled substance is necessary in internal signing and implementation of mobility skill. Therefore, a random practice plan with decreased frequency of feedback can directly create challenge in change of task and internal signing in Parkinson patients; abundant iterations of the task lead to learning(Onla-or \& Winstein, 2007). In this research, it seems that the outcome is resulted from improvement in cerebellum function due to practice protocol of stepping. We can also refer to the studies of Pollock et al (2014) that found results similar to the results of this research about usefulness of high demand practice conditions and 
increase in aimed task difficulty. They argued that application of movement learning principles was effective on retraining of stepping reaction to improve balance control of brain stroke patients(Pollock et al., 2014).

In a research by Rampello et al (2007), a four-week aerobic sport period caused a significant increase in speed and gait in MS patients (22). Motl et al (2012) investigated gait enhancing combined practice in MS patients. The combined practice significantly improved walking, gait speed and people movement (Motl et al., 2012). It can be said that implementation of sport program must be appropriate to the ability of MS patients so that they are able to undertake the tasks. In this research, we considered CPF and adjustment of difficulty level proportional to the abilities of the patients and physical aid in need. However, the results of the research by Dodd et al (2011) were not consistent with the results of this study. They conducted a review study about influence of resisting progressive practices on gait and increase in muscle ability in MS patients. They demonstrated that these practices were not effective on gait speed but caused increase in short-term resistance and decrease in fatigue. It was discussed that the reason for that the practices were not improved the gait was the type of selected practice and that the volume of the practices was not also consistent with those in other studies (Dodd et al., 2011). Given the results of this research and the familiarities with the MS patients, we found that decrease in mobility and general abilities cause increasing depression in the patients. Thus, the results of this research and other studies have demonstrated that physical activities appropriate to the abilities of the patients as an important and supplementary low-cost treatment can be helpful for the patients. It is clear that each plan must be appropriate to the needs of the patients to have its efficiency. The protocol of this research has particularly considered this issue. The limitation of this study was that we could not perform the method on women MS patients to examine the gender differences in applying the CPF. We suggest that future works can focus on the gender differences and long term following up after interference to identify the sustainability of the practice impacts for generalization.

\section{Conclusion}

Given the results of the study based on efficiency of CPF-based practices on mobility of MS patients, it can be recommended to apply this practice protocol as a new method in design and health planning to improve the situations of the MS patients. One of the goals of the coaches and physiotherapists is to formulate an appropriate practice program to increase Learning during practice. Thus, they may seek to find the best practice plan to enhance the Learning. However, in rehabilitation and practice treatment, the practice duration is limited. Hence, the therapists have to choose the practices to be able to make efficient movement Learning.

\section{References}

1. Akizuki, K., \& Ohashi, Y. (2013). Changes in practice schedule and functional task difficulty: a study using the 
probe reaction time technique. Journal of Physical Therapy Science, 25(7), 827-31. http://doi.org/10.1589/jpts.25.827

2. Bethoux, F., \& Bennett, S. (2011). Evaluating walking in patients with multiple sclerosis: which assessment tools are useful in clinical practice? International Journal of MS Care, 13(1), 4-14. http://doi.org/10.7224/15372073-13.1.4

3. Blair, T. C., Manoharan, M., Rawlings-Rhea, S. D., Tagge, I., Kohama, S. G., Hollister-Smith, J., Wong, S. W. (2016). Immunopathology of Japanese macaque encephalomyelitis is similar to multiple sclerosis. Journal of Neuroimmunology, 291, 1-10. http://doi.org/10.1016/j.jneuroim.2015.11.026

4. Dodd, K., Taylor, N. F., Shields, N., Prasad, D., McDonald, E., \& Gillon, A. (2011). Strength training can improve muscle performance, quality of life and fatigue in adults with multiple sclerosis: A randomised controlled trial. Physiotherapy (United Kingdom), 97, eS293-eS294.

http://doi.org/10.1016/j.physio.2011.04.002

5. Ghasemi, M., Gorji, Y., Ashtar, F., \& Ghasemi, M. (2015). A study of psychological well-being in people with multiple sclerosis and their primary caregivers. $A d v$ Biomed Res, 4, 49. http://doi.org/10.4103/22779175.151545

6. Guadagnoli, M. A., \& Bertram, C. P. (2014). Optimizing Practice for Performance Under Pressure From the Laboratory. International Journal of Golf Science, 2, 119-127. Retrieved from http://journals.humankinetics.com/doi/abs/10.1123/ijgs. 2014-0021

7. Guadagnoli, M., \& Aylsworth, B. (2013). Efficiency in Transfer from the Anchored to Standard Golf Putter. International Journal of Sports. Retrieved from http://journals.sagepub.com/doi/abs/10.1260/17479541.8.4.755

8. Guadagnoli, M., \& Lee, T. (2004). Challenge point: a framework for conceptualizing the effects of various practice conditions in motor learning. Journal of Motor Behavior. Retrieved from http://www.tandfonline.com/doi/abs/10.3200/JMBR.36. 2.212-224

9. Guadagnoli, M., \& Lindquist, K. (2007). Challenge Point Framework and Efficient Learning of Golf. International Journal of Sports Science and Coaching, 2, 185-197. http://doi.org/10.1260/174795407789705505

10. Hoang, P. D., Cameron, M. H., Gandevia, S. C., \& Lord, S. R. (2014). Neuropsychological, balance, and mobility risk factors for falls in people with multiple sclerosis: a prospective cohort study. Archives of Physical Medicine and Rehabilitation, 95(1532-821X (Electronic);-0003$9993 \quad$ (Linking)), 480. http://doi.org/10.1016/j.apmr.2013.09.017

11. Ilett, P., Lythgo, N., Martin, C., \& Brock, K. (2015).Intervention based on the bobath concept on balance and gait in people with multiple sclerosis. Physiotherapy Research, 101, 2015. Retrieved from http://onlinelibrary.wiley.com/doi/10.1002/pri.1624/pdf

12. Karlsson, A., \& Persson, T. (1997). The ankle strategy for postural control--a comparison between a model- based and a marker-based method. Comput.Methods Programs Biomed., 52(3), 165-173. Retrieved from http://www.sciencedirect.com/science/article/pii/S0169 260796017944

13. Krause, D., Brüne, A., Fritz, S., Kramer, P., Meisterjahn, P., Schneider, M., \& Sperber, A. (2014). Learning of a Golf Putting Task with Varying Contextual Interference Levels Induced by Feedback Schedule in Novices and Experts. Perceptual and Motor Skills, 118(2), 384-399. http://doi.org/10.2466/23.30.PMS.118k17w3

14. Lapierre, Y., O’Connor, P., Devonshire, V., Freedman, M. S., Kremenchutzky, M., Yeung, M., \& Schecter, R. (2016). Canadian experience with fingolimod: adherence to treatment and monitoring. Canadian Journal of Neurological Sciences/Journal Canadien des Sciences Neurologiques, 43(02), 278-283.

15. Martino, D., Espay, A., Fasano, A., \& Morgante, F. (2016). Abnormalities of Muscle Tone. Disorders of Movement. Retrieved from http://link.springer.com/chapter/10.1007/978-3-66248468-5_2

16. Motl, R. W., Smith, D. C., Elliott, J., Weikert, M., Dlugonski, D., \& Sosnoff, J. J. (2012). in Persons With Significant Disability From Multiple Sclerosis : A Pilot Study. Journal of Neurologic, 36(March), 32-37. http://doi.org/10.1097/NPT.0b013e3182477c92

17. Onla-or, S., \& Winstein, C. J. (2007). Determining the Optimal Challenge Point for Motor Skill Learning in Adults With Moderately Severe Parkinson's Disease. Neurorehabilitation and Neural Repair, 22(4), 385-395. http://doi.org/10.1177/1545968307313508

18. Peruzzi, A., Cereatti, A., Croce, U. Della, \& Mirelman, A. (2016). Effects of a virtual reality and treadmill training on gait of subjects with multiple sclerosis: A pilot study. Multiple Sclerosis and Related Disorders, 5, 91-96. http://doi.org/10.1016/j.msard.2015.11.002

19. Pollock, C. L., Boyd, L. A., Hunt, M. A., \& Garland, S. J. (2014). Use of the challenge point framework to guide motor learning of stepping reactions for improved balance control in people with stroke: a case series. Physical Therapy, 94(4), 562-70. http://doi.org/10.2522/ptj.20130046

20. Sebastia, E., Sandroff, B. M., Learmonth, Y. C., \& Motl, R. W. (2016). Validity of the Timed Up and Go Test as a Measure of Functional Mobility in Persons With Multiple Sclerosis. Archives of Physical, 1072-1077. http://doi.org/10.1016/j.apmr.2015.12.031

21. Verma, J. P. (2011). Statistical Methods for Sports and Physical Education. Retrieved from https://scholar.google.com/scholar?q=Statistical + metho $\mathrm{ds}+$ for + sports + and + physical + education $\& b \operatorname{bth}=\& \mathrm{hl}=\mathrm{en}$ \&as_sdt $=0 \% 2 \mathrm{C} 5$

22. Yim-Chiplis, P. K., \& Talbot, L. a. (2000). Defining and measuring balance in adults. Biological Research for Nursing, $1, \quad 321-331$. http://doi.org/10.1177/109980040000100408

23. Zampieri, C., \& Di Fabio, R. P. (2008). Balance and eye movement training to improve gait in people with progressive supranuclear palsy: quasi-randomized clinical trial. Physical Therapy, 88(12), 1460-73. http://doi.org/10.2522/ptj.20070302. 